\title{
Modeling Punching Shear Capacity of Fiber-Reinforced Polymer Concrete Slabs: A Comparative Study of Instance-Based and Neural Network Learning
}

\author{
Nhat-Duc Hoang, ${ }^{1}$ Duy-Thang Vu, ${ }^{2}$ Xuan-Linh Tran, ${ }^{1}$ and Van-Duc Tran ${ }^{3}$ \\ ${ }^{1}$ Institute of Research and Development, Faculty of Civil Engineering, Duy Tan University, K7/25 Quang Trung, Danang, Vietnam \\ ${ }^{2}$ Faculty of Architecture, Duy Tan University, K7/25 Quang Trung, Danang, Vietnam \\ ${ }^{3}$ International School, Duy Tan University, 254 Nguyen Van Linh, Danang 550000, Vietnam \\ Correspondence should be addressed to Nhat-Duc Hoang; hoangnhatduc@dtu.edu.vn
}

Received 1 December 2016; Revised 17 January 2017; Accepted 15 March 2017; Published 4 April 2017

Academic Editor: Lukasz Sadowski

Copyright (C) 2017 Nhat-Duc Hoang et al. This is an open access article distributed under the Creative Commons Attribution License, which permits unrestricted use, distribution, and reproduction in any medium, provided the original work is properly cited.

This study investigates an adaptive-weighted instanced-based learning, for the prediction of the ultimate punching shear capacity (UPSC) of fiber-reinforced polymer- (FRP-) reinforced slabs. The concept of the new method is to employ the Differential Evolution to construct an adaptive instance-based regression model. The performance of the proposed model is compared to those of Artificial Neural Network (ANN) and traditional formula-based methods. A dataset which contains the testing results of FRP-reinforced concrete slabs has been collected to establish and verify new approach. This study shows that the investigated instance-based regression model is capable of delivering the prediction result which is far more accurate than traditional formulas and very competitive with the black-box approach of ANN. Furthermore, the proposed adaptive-weighted instanced-based learning provides a means for quantifying the relevancy of each factor used for the prediction of UPSC of FRP-reinforced slabs.

\section{Introduction}

In civil engineering, fiber-reinforced polymer (FRP) composites have been increasingly employed due to their strength and stiffness, good thermomechanical properties, capacity for resisting corrosion, low weight, and outstanding durability [1-4]. It is proper to note that corrosion of steel reinforcement is the major factor which influences the deterioration and shortens the service life of reinforced concrete structures [5-7]. Thus, the utilizations of FRP composites have created the condition for enhancing the productivity of construction process, meliorating the performance of concrete structures, reduction of maintenance budgets spent for infrastructure, and possible elongation of structure service lives $[8,9]$.

Steel-reinforced two-way flat slabs are popular structural systems that can simplify and accelerate on-site operations and facilitate flexible partitioning of space [10]. The two-way flat slabs are particularly efficient for constructing parking structures. Additionally, computing the slab's resistance of shear stresses at supporting columns is a major concern in the design procedure of this structure [11]. Notably, the slab-supporting column connections have been shown to be vulnerable to high shear stresses and this might bring about brittle and sudden punching shear failures [10]. Especially when the steel reinforcements get corroded due to moisture and other hostile factors in the operational environment, punching shear failures may occur at these slab-column connections. Accordingly, these may lead to progressive collapse of the whole structure [12].

Due to such reasons, FRP bars are recently considered as effective substitutions to the traditional steel bars in concrete flat slabs [13]. Moreover, UPSC is critical factor which determines the design process of concrete slabs supported by columns. Thus, this problem is extensively studied in the literature [14]. As a consequence, various researches have been conducted to investigate the applicability of existing 
empirical approaches and modify them to predict the punching shear capacity of FRP-reinforced slabs $[1,10,15,16]$.

Recently, machine learning has been proved to provide a feasible alternative for modeling the punching shear capacity of FRP-reinforced slabs $[6,9,15,17]$. The Artificial Neural Network (ANN) has been employed to predict the FRPreinforced slab punching shear capacity [6]. The research shows that the predictive result produced by ANN is considerably more accurate than those computed by the empirical formulas. Despite the fact that ANN is a powerful method for modeling nonlinear systems $[18,19]$, the learning process of ANN suffers from certain challenges [20].

One major difficulty of ANN is that its model establishment stage is accomplished via a gradient descent (GD) algorithm. GD algorithm is known to be very complex and may contain many local minima [20]. Another disadvantage of the ANN approach lies in their knowledge representation [21]; the black-box nature of the method makes it difficult for structural engineers to comprehend how the ANN predicts the punching shear capacity of FRP-reinforced slabs. In addition, the ANN approach intrinsically provides no means of measuring the contribution of each input factors to the model performance.

This research aims at extending the body of knowledge by investigating a new learning alternative for modeling the punching shear capacity of FRP-reinforced slabs. The proposed approach is hybridization of an improved kernel regression and the Differential Evolution (DE) [22]. The research objective is to establish a prediction model with transparent structure, self-adaptive learning, and the capability to express the relevancy of input variables.

Notably, the kernel regression belongs to the class of instance-based regression, which is also called nonparametric regression. Nonparametric regression offers a flexible and effective way of approximating the regression function especially when the form of the regression function is inherently complex $[23,24]$. The reason is that, in such circumstance, it can be difficult to construct a universal parametric model based on a limited amount of training samples. Since good performances of the nonparametric approach have been observed throughout the literature [21, 25-28], the nonparametric regression is worth being investigated in solving the problem of interest.

Furthermore, learning based on instances or examples is a common practice in construction engineering. Thus, an instance-based model for punching shear capacity of FRP-reinforced slabs can be easily perceived by practical engineers; this may facilitate the applicability of the new approach. The rest of the article is organized as follows. The second section introduces the research method. The third section describes the proposed hybrid instance-based learning, followed by the experimental results. The final section summarizes our research with several conclusions.

\section{Research Methodology}

2.1. A Review of Formulas for Estimating Punching Shear Capacity of FRP-Reinforced Slabs. The shear resistance of the concrete $\left(V_{c}\right)$ is known to influence the punching shear capacity of two-way reinforced concrete flat slabs. $V_{c}$ acts over the area proportional to the length of a critical perimeter $\left(b_{o}\right)$ multiplied by the effective depth of the section $(d)$. Currently, there is a critical need to investigate design equations and prediction models for determining the punching shear strength of concrete slabs reinforced with FRP composite bars. The reason is that existing design equations applied for FRP-reinforced concrete sections are originated from those previously applied for steel-reinforced counterparts with certain adjustments for considering the replacement of steel by FRP. The following section reviews formula-based methods for the prediction of UPSC of FRP-reinforced slabs; it is noted that the system of units for all equations is SI.

The American Concrete Institute (ACI) Code (ACI 31811) introduces a design formula to account for the shear transfer in two-way steel-reinforced concrete slabs:

$$
V_{c}=0.33 \sqrt{f_{c}^{\prime}} b_{0,0.5 d} d
$$

where $f_{c}^{\prime}$ denotes the specified compressive strength of the concrete $(\mathrm{MPa}), b_{0,0.5 d}$ represents the perimeter of the critical section for slabs and footings at a distance of $d / 2$ away from the column face, and $d$ is the average flexural depth of the slab.

In addition, The British Standard (BS 8110-97) suggests a formula to attain the punching shear capacity for steelreinforced slabs as shown below:

$$
V_{c}=0.79\left(100 \rho_{s}\right)^{1 / 3}\left(\frac{400}{d}\right)^{1 / 4}\left(\frac{f_{\mathrm{cu}}}{25}\right)^{1 / 3} b_{0,1.5 d} d
$$

where $f_{\text {cu }}$ denotes the characteristic concrete cube compressive strength $\left(\mathrm{N} / \mathrm{mm}^{2}\right), \rho_{s}$ represents the steel reinforcement ratio, $b_{0,1.5 d}$ is the perimeter of the critical section for slabs and footings at a distance of $1.5 \mathrm{~d} / 2$ away from the loaded area $(\mathrm{mm})$, and $d$ represents the average flexural depth of the slab.

On the basis of experiments, El-Ghandour et al. [29] suggested a modification to the ACI's equation by multiplying it by the term $\left(E_{f} / E_{s}\right)^{1 / 3}$ to account for the use of FRP bars as follows:

$$
V_{c}=0.33 \sqrt{f_{c}^{\prime}}\left(\frac{E_{f}}{E_{s}}\right)^{1 / 3} b_{0,0.5 d} d
$$

where $E_{f}$ and $E_{s}$ are Young's modulus of the FRP-reinforced slab and Young's modulus of the steel reinforcement, respectively.

El-Ghandour et al. [30] modified the BS 8110-97's design equation and suggested an alternative formula to obtain the shear strength of FRP-reinforced concrete slabs as follows:

$$
\begin{aligned}
V_{c}= & 0.79\left(100 \rho_{s} 1.8\left(\frac{E_{f}}{E_{s}}\right)\right)^{1 / 3}\left(\frac{400}{d}\right)^{1 / 4}\left(\frac{f_{\mathrm{cu}}}{25}\right)^{1 / 3} \\
& \cdot b_{0,1.5 d} d .
\end{aligned}
$$


TABLE 1: Statistical descriptions of variables.

\begin{tabular}{|c|c|c|c|c|c|}
\hline Input factors & Notation & Min & Average & Std. Dev. & Max \\
\hline The type of column section & $X_{1}$ & - & - & - & - \\
\hline Section area of column $\left(\mathrm{mm}^{2}\right)$ & $X_{2}$ & 5027.0 & 76203.6 & 56353.8 & 202500.0 \\
\hline Effective flexural depth of slab (mm) & $X_{3}$ & 55.0 & 141.4 & 61.9 & 284.0 \\
\hline Compressive strength of concrete ( $\mathrm{MPa})$ & $X_{4}$ & 26.0 & 41.7 & 13.1 & 118.0 \\
\hline Young's modulus of the FRP slab (GPa) & $X_{5}$ & 28.4 & 67.4 & 32.8 & 147.6 \\
\hline Reinforcement ratio (\%) & $X_{6}$ & 0.2 & 0.9 & 0.7 & 3.8 \\
\hline Punching shear capacity $(\mathrm{kN})$ & Y & 61.0 & 470.7 & 360.6 & 1600.0 \\
\hline
\end{tabular}

Matthys and Taerwe [31] put forward an enhancement of the BS 8110-97 as follows:

$$
V_{c}=1.36\left(\frac{100 \rho_{s}\left(E_{f} / E_{s}\right) f_{c}^{\prime}}{d^{1 / 4}}\right)^{1 / 3} b_{0,1.5 d} d .
$$

Ospina et al. [32] introduced an improved version of the equation proposed by Matthys and Taerwe [31]; in this revision, the cube root of the modular ration is replaced by the square root. This design formula is shown as follows:

$$
V_{c}=2.77\left(\rho_{s} f_{c}^{\prime}\right)^{1 / 3} \sqrt{\frac{E_{f}}{E_{s}}} b_{0,1.5 d} d .
$$

A design equation has been proposed by the subcommittee ACI440H [16] for calculation of steel-reinforced two-way concrete slabs. This equation has considered the influence of reinforcement stiffness to account for the shear transfer in two-way concrete slabs as follows:

$$
V_{c}=0.8 \sqrt{f_{c}^{\prime}} b_{0,0.5 d} c
$$

where $b_{0,0.5 d}$ is the perimeter of the critical section for slabs and footings at a distance of $d / 2$ away. And $c$ is the cracked transformed section neutral axis depth $(\mathrm{mm})$ and is calculated as follows:

$$
c=k \cdot d,
$$

where $k$ is defined in the following equation:

$$
k=\sqrt{2 \rho_{f} n_{f}+\left(\rho_{f} n_{f}\right)^{2}}-\rho_{f} n_{f} .
$$

In (9), it is noted that $n_{f}=E_{f} / E_{c}$ denotes modular ratio and $E_{c}=4700 \sqrt{f_{c}^{\prime}}$ is the concrete modulus of elasticity.

2.2. The Collected Dataset of Punching Shear Tests. The dataset of FRP-reinforced concrete flat slab used in this study consists of 82 tests recorded in previous research works [6, $15,33]$. As the previous work of $\mathrm{Vu}$ and Hoang [17], the type of column section $\left(X_{1}\right)$, section area of column $\left(X_{2}\right)$, effective flexural depth of slab $\left(X_{3}\right)$, compressive strength of concrete $\left(X_{4}\right)$, Young's modulus of the FRP-reinforced slab $\left(X_{5}\right)$, and reinforcement ratio $\left(X_{6}\right)$ are considered as input factors that determine the ultimate punching capacity of the FRP-reinforced concrete flat slab. The shape of the column section includes three forms: square $\left(X_{1}=1\right)$, circle $\left(X_{1}=2\right)$, and rectangle $\left(X_{1}=3\right)$. In the dataset, the numbers of square, circle, and rectangle sections are 50,13, and 19, respectively. The range of the punching capacity varies from $61 \mathrm{kN}$ to $1600 \mathrm{kN}$. Table 1 shows the variables and their statistical descriptions. The whole dataset is provided in the Appendix.

2.3. Instance-Based Regression. Given a set of independent observations $\left(x_{i}, y_{i}\right)_{i=1, \ldots, N}$, where $X=\left\{x_{i}\right\}$ and $Y=\left\{y_{i}\right\}$ are called predictor variable and response variable, respectively, and $N$ denotes the number of data points, the task of regression analysis is to construct a function $m(x)$ that provides the mapping relation between the predictor variable and the response variable:

$$
y_{i}=m\left(x_{i}\right)+\varepsilon_{i},
$$

where $\varepsilon_{i}$ represents a random noise. $m\left(x_{i}\right)$ is generally called the regression function or regression of $Y$ on $X$, which can be employed to predict the value of $Y$ based on an unknown input variable $X$.

The approaches of regression analysis can be categorized into two major classes: parametric model and nonparametric model [34]. In the former class, the underlying functional form of the model is known and the model's free parameters are estimated directly from the dataset at hand. Linear/nonlinear regression models are the typically parametric and the Artificial Neural Network (ANN) can be considered as an advanced form of nonlinear parametric model. In the latter class, there is no restriction on the functional form of the regression model; the model generally stores all the collected data instances in its memory and utilizes such memory to predict unknown input data [24, 35]. Therefore, the approach of nonparametric learning is also called instance-based learning.

Previous studies found that the restriction of $m(x)$ to a certain parametric form can limit the flexibility of the learning capability. A particularly undesirable situation happens when a parametric model that is inappropriate for the dataset is chosen. In such case, the model in no way can reflect the nature of the dataset regardless of how accurately its free parameters are estimated. Furthermore, this shortcoming 
of parametric models can be circumvented by abandoning the restriction on the functional form of $m(x)$. This leads to the method of nonparametric learning or instance-based learning.

Instance-based learning offers an approach for constructing the mapping function with a greater degree of flexibility [24]. This approach is particularly useful when the underlying form of the regression function is very complex. In such a case, it is much more efficient to construct a series of simple local regression models instead of a complicated global regression model. Furthermore, a significant advantage of instance-based learning is that its prediction process can be easily comprehended by practical engineers; this is in contrast to the black-box learning approach used in the ANN.

In this research, the instance-based learning approach of interest is the kernel regression with the utilization of kernel smoothing. In the kernel regression, the estimated value of the response variable $y$ is computed by a weighted average of all the data stored in the model's memory. The predicted value $y$ of the predictor variable $x$, denoted as $y_{p}$, is computed as follows [36-38]:

$$
y_{p}=\frac{\sum_{i=1}^{N} \theta_{i} \times y_{i}}{\sum_{i=1}^{N} \theta_{i}}
$$

where $y_{i}$ represents the $i$ th data point in the memory which consists of $N$ data points.

The Gaussian kernel is commonly selected to calculate the weight $\theta_{i}$ as follows:

$$
\theta_{i}=\frac{1}{\sqrt{2 \pi} \times h} \exp \left[\frac{-d\left(x, x_{i}\right)^{2}}{2 \times h^{2}}\right]
$$

where the parameter $h$ denotes the kernel width and $d\left(x, x_{i}\right)$ is the similarity between the two data points $x$ and $x_{i}$.

Herein, the Minkowski distance, a generalization of both the Euclidean distance and the Manhattan distance, is used to measure the similarity between data points:

$$
d\left(x, x_{i}\right)=\left(\sum_{k=1}^{D}\left|x_{k}-x_{i, k}\right|^{q}\right)^{1 / q}
$$

where $D$ is the data dimension and $q \in[1, \infty)$ is a scalar. It is noted that when $q=2$ and $q=1$, the Minkowski distance is equivalent to the Euclidean distance and Manhattan distance, respectively.

In addition, it is noted that the relevance of each input factor, or feature, can be quantified by associating it with a weighting value which varies from 0 to 1 . The higher the weighting value is, the more relevant the input attribute is. The feature weights can be embedded into the distance function to measure the similarity between two data instances. The incorporation of feature weight into the instance-based regression model and more detailed explanation are discussed in Section 3 of the article.

2.4. Differential Evolution (DE). DE is an evolutionary algorithm developed by Storn and Price [22]. This metaheuristic is a population-based stochastic approach which is very effective for global optimization in continuous domains $[39,40]$. The DE algorithm relies on a novel crossovermutation operator which is a linear combination of three different individuals and one subject-to-replacement parent (also called target vector) [41, 42]. This crossover-mutation operator of the DE algorithm aims at producing a trial vector (also called child vector) which must compete with its parent through the selection process. The selection operator of DE is a fitness based comparison between the parent and the corresponding offspring [43].

The DE algorithm [22] is unquestionably one of the most powerful approaches for solving complex optimization problems $[44,45]$. Successful applications of the DE optimization algorithm as well as other metaheuristic approaches for solving complex or ill-defined engineering problems have been observed in various fields [46-51]. Given that the problem of interest is to minimize a cost function $f(\mathbf{X})$, where the number of decision variables is $D$, the $\mathrm{DE}$ algorithm can be described in Algorithm 1. In Algorithm 1, it is noted that $\operatorname{rnd}(i)$ denotes a random integer.

Initially, a population consisting of $D$-dimensional parameter vectors $X_{i, g}$ is randomly generated, where $i=1,2, \ldots, N P$ and $g$ denotes the current generation number. Accordingly, a mutant vector is computed for each target vector in the following manner:

$$
V_{i, g+1}=X_{r 1, g}+F \times\left(X_{r 2, g}-X_{r 3, g}\right),
$$

where $r 1, r 2$, and $r 3$ are random indexes lying between 1 and NP. NP represents the population size. $F$ denotes the mutation scale factor, which controls the amplification of the differential variation. $V_{i, g+1}$ represents the newly created mutant vector.

The crossover operator of DE aims at increasing the diversity of the current population by exchanging components of the target and mutant vectors. Within the crossover phase, a trial vector is generated in the following way:

$$
U_{j, i, g+1}= \begin{cases}V_{j, i, g+1}, & \text { if } \operatorname{rand}_{j} \leq \operatorname{Cr} \text { or } j=\operatorname{rnb}(i) \\ X_{j, i, g}, & \text { if } \operatorname{rand}_{j}>\operatorname{Cr} \text { and } j \neq \operatorname{rnb}(i),\end{cases}
$$

where $U_{j, i, g+1}$ represents the trial vector. $j$ denotes the index of element for any vector. rand $_{j}$ is a uniform random number lying between 0 and 1 . Cr denotes the crossover probability. $\mathrm{rnb}(i)$ represents a randomly chosen index of $\{1,2, \ldots, N P\}$ which guarantees that at least one parameter from the mutant vector $\left(V_{j, i, g+1}\right)$ is copied to the trial vector $\left(U_{j, i, g+1}\right)$.

The selection stage compares the trial vector with the target vector to identify a better solution for the optimization problem at hand. The selection operator is demonstrated as follows:

$$
X_{i, g+1}= \begin{cases}U_{i, g} & \text { if } f\left(U_{i, g}\right) \leq f\left(X_{i, g}\right) \\ X_{i, g} & \text { if } f\left(U_{i, g}\right)>f\left(X_{i, g}\right)\end{cases}
$$

2.5. Artificial Neural Network (ANN). ANN is a soft computing method which is inspired from biological neural networks. This technique simulates the knowledge acquisition 


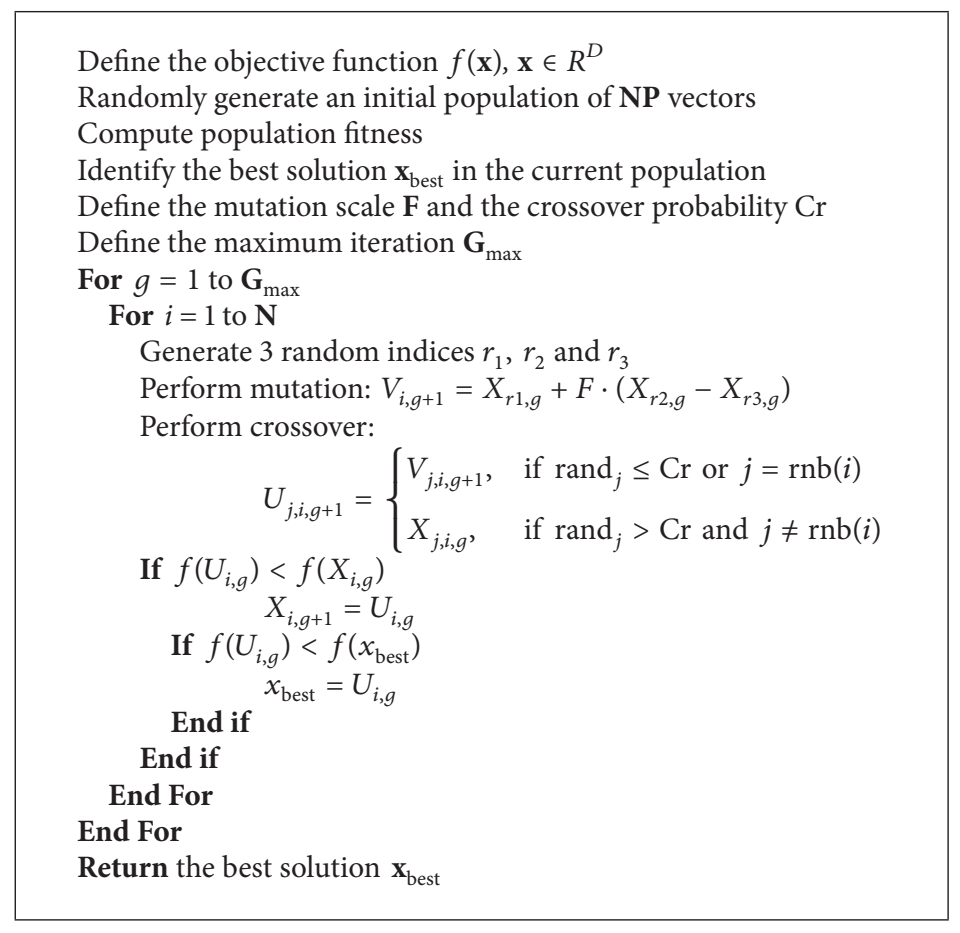

Algorithm 1: Differential Evolution (DE).

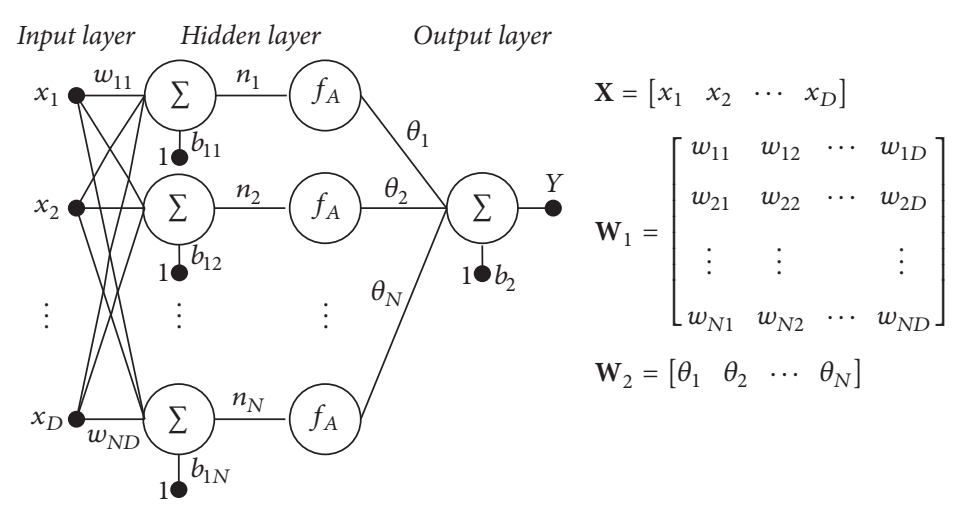

Figure 1: Artificial Neural Network structure.

and inference processes in the human brain. The advantages of the ANN can be realized by its flexibility and universal learning capability. Nevertheless, primary drawbacks of the ANN are the trial-and-error process for properly determining a network configuration and its black-box learning nature.

The learning task is to train a function $f: X \in R^{D} \rightarrow$ $Y \in R^{1}$, where $D$ denotes the number of input attributes. An ANN model, which includes the input, hidden, and output layers, is demonstrated in Figure $1 . W_{1}$ and $W_{2}$ represent weight matrices of the hidden layer and the output layer, respectively; $N$ denotes the number of neurons in the hidden layer; $b_{1}=\left[b_{11}, b_{12}, \ldots, b_{1 N}\right]$ represents a bias vector of the hidden layer; $b_{2}$ is a bias vector of the output layer; $f_{A}$ denotes an activation function (e.g., log-sigmoid).
The ANN structure used for regression analysis is shown as follows [52]:

$$
f(X)=b_{2}+W_{2} \times\left(f_{A}\left(b_{1}+W_{1} \times X\right)\right) .
$$

Generally, the weight matrices and the bias vectors of an ANN are trained via a process that employs the framework of error backpropagation [19]. Moreover, the Mean Square Error (MSE) is employed as the objective function for training an ANN structure for function approximation tasks [53]:

$$
\mathrm{MSE}=\min _{W_{1}, W_{2}, b_{1}, b_{2}} \frac{1}{M} \sum_{i=1}^{M} e_{i}^{2},
$$




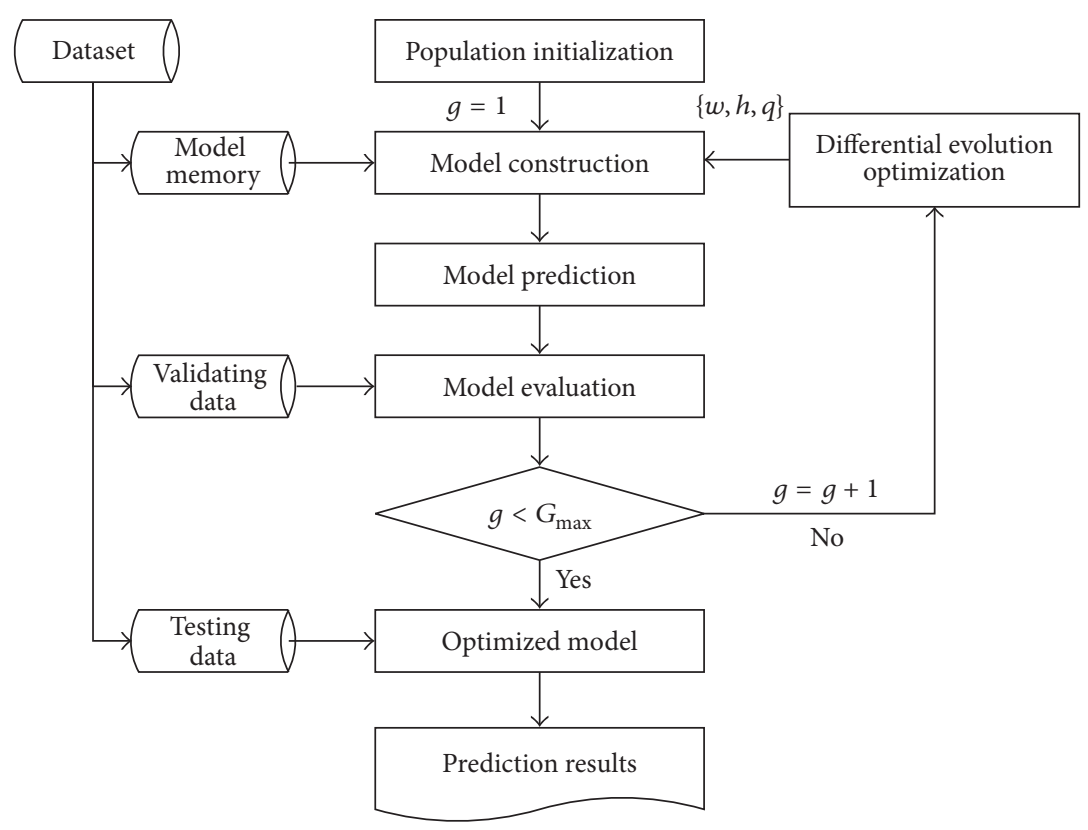

FIGURE 2: The proposed instance-based regression model for the prediction of punching shear capacity of FRP-reinforced concrete slabs.

where $M$ represents the number of data samples and $e_{i}$ is an output error. $e_{i}=Y_{i, P}-Y_{i, A}\left(Y_{i, P}\right.$ and $Y_{i, A}$ denote the predicted and actual outputs, resp.).

\section{The Proposed Adaptive-Weighted Instance-Based Regression Model for the Prediction of Punching Shear Capacity of FRP-Reinforced Concrete Slabs}

This section of the article describes the proposed instancebased regression model for the prediction of punching shear capacity of FRP-reinforced concrete slabs, denoted as IRMPSC in detail. The main idea of the IRM-PSC is to employ the DE optimization algorithm to select an appropriate set of the instance-based regression model's parameters including the kernel width $(h)$, the Minkowski distance scalar $(q)$, and the feature weights $\left(w_{k}\right)$. The proposed model consists of five major steps: data processing, model construction and model prediction, Differential Evolution optimization, model evaluation, and optimized model. Figure 2 illustrates the IRM-PSC's flowchart. In this figure, $g$ and $G_{\max }$ represent the current generation and the maximum generation of the DE algorithm, respectively.

3.1. Data Processing. The dataset including 82 punching tests of FRP-reinforced concrete slab collected from the literature is employed to construct and verify the prediction model. The type of column section, section area of column, effective flexural depth of slab, compressive strength of concrete, Young's modulus of the FRP-reinforced slab, and reinforcement ratio are considered as influencing factors that determine the ultimate punching capacity of the FRPreinforced concrete flat slab. The whole dataset is randomly separated into three sets: the training, validating, and testing sets. The training set is used to establish the model memory; the other two datasets are employed to validate and test the model performance. It is noted that, before being separated, the whole dataset has been normalized into the range of $[0,1]$. This data normalization aspires to prevent the situation in which features with greater numeric magnitudes dominate those with smaller magnitudes.

3.2. Model Construction and Model Prediction. At this phase, the kernel regression is utilized as the instance-based learning approach to model the mapping relation between the ultimate punching capacity of the FRP-reinforced concrete flat slab and its six influencing input variables. The model simply stores all data samples of the training set in its memory. When new input data is available, the model computes the kernel weights using this new sample and other existing samples. Accordingly, the estimated punching capacity of the new input data is calculated as a weighted average of all punching capacities of all the data in the memory.

Furthermore, it is noted that, in this study, the relevance of the input feature $k$ is quantified by its feature weight $w_{k}$ varying from 0 to 1 . As mentioned earlier, a higher feature weight means that the feature of interest is more relevant for estimating the output. The feature weights are embedded into the distance measurement between two data instances. Accordingly, the modified Minkowski distance is provided as follows:

$$
d\left(x, x_{i}\right)=\left(\sum_{k=1}^{D} w_{k} \times\left|x_{k}-x_{i, k}\right|^{q}\right)^{1 / q}
$$

It is worth noticing that each data feature represents an axis in the input space. For the problem at hand, the input 
TABLE 2: Prediction performance of the IRM-PSC and ANN models.

\begin{tabular}{lcccc}
\hline & & RMSE & MAPE (\%) & $R^{2}$ \\
\hline \multirow{2}{*}{ IRM-PSC } & Training & 34.62 & 1.22 & 0.99 \\
& Testing & 63.34 & 12.70 & 0.96 \\
\multirow{2}{*}{ ANN } & Training & 64.29 & 17.14 & 0.97 \\
& Testing & 52.43 & 15.98 & 0.96 \\
\hline
\end{tabular}

space is a six-dimensional space. As can be seen from (19), the introduction of the feature weight $w_{k}$ can elongate or truncate the axis $k$ in the input space. A high $w_{k}$ elongates the axis $k$; this makes the feature $k$ very significant for the prediction process. The reason is that when the axis is elongated, even a small difference of $\left|x_{k}-x_{i, k}\right|$ can result in a large kernel weight. The reversed result occurs when an axis is truncated. In the extreme case when $w_{k}=0$, the quantity $\left|x_{k}-x_{i, k}\right|$ imposes no influence on the final kernel weight between two data instances. It is noted that the feature weight $w_{k}$ used in this section is different from the Gaussian kernel weight $\left(\theta_{i}\right)$ defined in (11) and (12). The feature weight expresses the relevancy of input variable; meanwhile, the Gaussian kernel weight exhibits the data similarity.

3.3. Differential Evolution Optimization. The construction of the model necessitates the kernel width $(h)$, the Minkowski distance scalar $(q)$, and the feature weights $\left(w_{k}\right)$ as hyperparameters. At each iteration, the $\mathrm{DE}$ algorithm guides the population of vectors to search for better solutions. Gradually, inferior solutions characterized with worse values of fitness are discarded; superior solutions with better values of fitness survive to the next iterations. The DE's search progresses until the current generation $(g)$ exceeds the maximum generation $\left(G_{\max }\right)$.

3.4. Model Evaluation. To identify an appropriate set of hyperparameters, it is necessary to define an objective function that quantifies the model performance. In this study, the model's prediction accuracy in terms of Root Mean Squared Error (RMSE) when estimating the validating dataset is employed for model evaluation:

$$
f=\sqrt{\sum_{j=1}^{N} \frac{\left(Y_{P}^{j}-Y_{A}^{j}\right)^{2}}{N}},
$$

where $Y_{P}^{j}$ and $Y_{A}^{j}$ denote predicted and actual value for output $j$ th. In addition, $N$ is the number of data samples in the validating set.

3.5. Optimized Model. When the DE based searching process terminates, the optimized prediction model, characterized by an appropriate set of hyperparameters, has been identified. The proposed IRM-PSC is ready for the prediction of UPSC of the FRP-reinforced concrete flat slab.

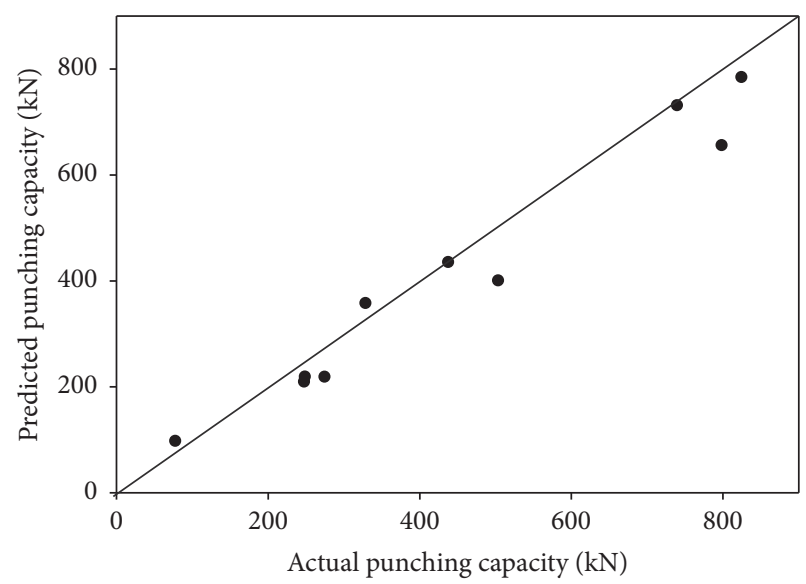

FIGURE 3: Testing results of the proposed IRM-PSC $\left(R^{2}=0.96\right)$.

\section{Experimental Results}

When the IRM-PSC model has been constructed, the proposed model can be employed to predict the punching shear capacity of FRP-reinforced flat slab. Besides the proposed instance-based regression, previously used methods including ANN [6] and the empirical formulas are utilized as benchmark approaches. The ANN consists of 1 hidden layer with 6 neurons. The applied learning rate was 0.001 together with a number of 5000 learning epochs. The LevenbergMarquardt algorithm is used to train the ANN model [54]. Additionally, to quantify model performance, Root Mean Squared Error (RMSE), Mean Absolute Percentage Error (MAPE), and Coefficient of Determination $\left(R^{2}\right)$ are computed.

In the first experiment, the dataset has been randomly divided into two sets; the first set (Set 1) was used for model construction, while the second set (Set 2) was used for model testing. The numbers of data samples in Set 1 and Set 2 are 72 and 10, respectively. Set 1 is further divided into two subsets: subset 1 which constitutes the model memory (80\%) and subset $2(20 \%)$ which is reserved for model validating. The testing results of the IRM-PSC are illustrated in Figure 3. This figure demonstrates that the proposed approach has obtained a good fit to a straight line.

Furthermore, in this experiment, the prediction performance of the proposed instance-based model is compared to that of the ANN. The results comparison is reported in Table 2. The RMSE, MAPE, and $R^{2}$ in the testing process 
TABLE 3: Result comparison.

\begin{tabular}{lccccccccc}
\hline $\begin{array}{l}\text { Prediction } \\
\text { methods }\end{array}$ & IRM-PSC ANN & $\begin{array}{c}\text { ACI } \\
318-11\end{array}$ & $\begin{array}{c}\text { BS } \\
8110-97\end{array}$ & $\begin{array}{c}\text { El-Ghandour } \\
\text { et al. (1999) }\end{array}$ & $\begin{array}{c}\text { El-Ghandour } \\
\text { et al. (2000) }\end{array}$ & $\begin{array}{c}\text { Matthys } \\
\text { and Taerwe } \\
(2000)\end{array}$ & $\begin{array}{c}\text { Ospina } \\
\text { et al. } \\
(2003)\end{array}$ & $\begin{array}{c}\text { ACI } \\
440 H\end{array}$ \\
\hline RMSE & $\mathbf{6 5 . 9 9}$ & $\mathbf{6 2 . 2 9}$ & 196.61 & 158.38 & 188.94 & 151.27 & 201.58 & $\mathbf{1 1 7 . 5 1}$ & 342.52 \\
MAPE & $\mathbf{1 1 . 8 1}$ & $\mathbf{1 2 . 8 6}$ & 28.88 & 28.02 & 28.86 & 17.07 & 24.13 & $\mathbf{1 5 . 4 8}$ & 56.02 \\
$R^{2}$ & $\mathbf{0 . 9 7}$ & $\mathbf{0 . 9 6}$ & 0.80 & 0.81 & 0.85 & 0.90 & 0.90 & $\mathbf{0 . 9 1}$ & 0.88 \\
\hline
\end{tabular}

obtained from the IRM-PSC are 63.34, 12.70, and 0.96. The ANN approach yields the following results: $\mathrm{RMSE}=52.43$, MAPE $=15.98$, and $R^{2}=0.96$. Observably, in terms of the MAPE, the new method has achieved 3.28\% improvement compared with the ANN. The two prediction models obtain the same value of $R^{2}$. Nevertheless, the ANN shows better performance in terms of RMSE.

In the second experiment, a tenfold cross validation process is conducted. Because all of the subsamples are mutually exclusive, the cross validation process can accurately evaluate the model performance. The average prediction performances (measured in RMSE, MAPE, and $R^{2}$ ) of the IRM-PSC and the ANN are reported in Table 3. Additionally, the prediction results obtained from formulabased approaches are also provided in this table. The results of the empirical formulas are calculated using the equations (from (1) to (7)) mentioned previously in this article.

From Table 3, it is recognizable that the equation proposed by Ospina et al. [32] is the best formula-based method, and the RMSE, MAPE, and $R^{2}$ of this method are 117.51, 15.48, and 0.91 , respectively. This outcome is shown to be far worse than the two machine learning approaches. RMSE, MAPE, and $R^{2}$ of the IRM-PSC are $65.99,11.81$, and 0.97 , respectively. The three measurements of the ANN are 62.29, 12.86, and 0.96 . Thus, the newly proposed method overcomes the ANN as measured by MAPE and $R^{2}$; meanwhile the ANN is better than the IRM-PSC in terms of RMSE. In summary, compared with the best empirical formula, the IRM-PSC has attained roughly $43 \%$ and $24 \%$ improvements in RMSE and MAPE, respectively.

Furthermore, it is beneficial to investigate the relevance of the six input features employed in this study. As mentioned earlier, the relevance of input features can be quantified by the feature weight $w_{k}$. The average feature weights of the six input attributes, obtained from the tenfold cross validation process, are as follows: $w_{1}=0.63, w_{2}=0.28, w_{3}=0.81, w_{4}=0.36$, $w_{5}=0.26$, and $w_{6}=0.76$. Thus, it can be seen that the type of column section $\left(X_{1}\right)$, the effective flexural depth of slab $\left(X_{3}\right)$, and the reinforcement ratio $\left(X_{6}\right)$ are highly relevant for the prediction of UPSC of the FRP-reinforced concrete slabs. The reason is that these three features obtain the highest feature weights. Meanwhile, the other input attributes, including the section area of column $\left(X_{2}\right)$, the compressive strength of concrete $\left(X_{4}\right)$, and Young's modulus of the FRP slab $\left(X_{5}\right)$, obtain lower values of feature weights. Nevertheless, since all weights are greater than zero, these three features $\left(X_{2}, X_{4}\right.$, and $\left.X_{5}\right)$ are still relevant for the prediction process.

\section{Conclusion}

This research proposed an alternative, named as IRM-PSC, for calculating the punching shear capacity of the FRPreinforced two-way slabs. The new approach employs the kernel regression as an instance-based learning technique to infer the mapping function between the punching shear capacity and its input features. To construct the instancebased learning model, the DE is employed.

Experimental results have proved that the IRM-PSC can deliver prediction performance which is far more accurate than commonly used empirical formulas, reflected in low prediction errors (RMSE and MAPE) and a high Coefficient of Determination $\left(R^{2}\right)$. The new method has also demonstrated a competitive capability compared with the ANN approach. However, as an instance-based learning method, the frameworks of learning and making predictions of the IRM-PSC are much more transparent than the black-box ANN. Furthermore, another advantage of the new method is that it can help to reveal the relevance of input features quantified by the feature weight. Thus, the new model can be better understood and easily applied by structural engineers, who may not be familiar with machine learning, to design FRP-reinforced slabs.

Thus, the applicability of the new approach may be limited to the ranges of input features recorded in the dataset. Accordingly, more slab test results should be collected both by actual experiments and by analyses via nonlinear Finite Element Method packages; these data can be incorporated into the current dataset to enhance the generalization and applicability of the IRM-PSC. These tasks can be a future direction of the current study.

\section{Appendix}

See Table 4.

\section{Conflicts of Interest}

The authors declare that there are no conflicts of interest regarding the publication of this article. 
TABLE 4: The dataset of punching shear tests.

\begin{tabular}{|c|c|c|c|c|c|c|}
\hline $\mathrm{X}_{1}$ & $\begin{array}{c}\mathbf{X}_{2} \\
\left(\mathrm{~mm}^{2}\right)\end{array}$ & $\begin{array}{c}\mathbf{X}_{3} \\
(\mathrm{~mm})\end{array}$ & $\begin{array}{c}\mathbf{X}_{4} \\
(\mathrm{MPa})\end{array}$ & $\begin{array}{c}\mathbf{X}_{5} \\
(\mathrm{GPa})\end{array}$ & $\begin{array}{l}\mathbf{X}_{6} \\
(\%)\end{array}$ & $\begin{array}{c}\mathbf{Y} \\
(\mathrm{kN})\end{array}$ \\
\hline 1 & 10000 & 55 & 52.9 & 100 & 0.31 & 61 \\
\hline 1 & 10000 & 55 & 41 & 100 & 0.31 & 65 \\
\hline 1 & 5625 & 61 & 44.6 & 113 & 0.95 & 78 \\
\hline 1 & 5625 & 61 & 42.4 & 113 & 0.95 & 93 \\
\hline 1 & 10000 & 61 & 39 & 113 & 0.95 & 96 \\
\hline 1 & 10000 & 61 & 36.6 & 113 & 0.95 & 99 \\
\hline 1 & 40000 & 82 & 37.4 & 46 & 1.1 & 165 \\
\hline 1 & 40000 & 142 & 33.3 & 45 & 0.22 & 170 \\
\hline 1 & 40000 & 112 & 33 & 46 & 0.81 & 170 \\
\hline 1 & 40000 & 129 & 39 & 48 & 0.48 & 180 \\
\hline 1 & 62500 & 120 & 37.5 & 28.4 & 0.87 & 206 \\
\hline 1 & 62500 & 100 & 26 & 42 & 0.95 & 210 \\
\hline 1 & 40000 & 82 & 38.2 & 46 & 1.29 & 210 \\
\hline 1 & 40000 & 129 & 39 & 48 & 0.68 & 212 \\
\hline 1 & 62500 & 120 & 29.5 & 34 & 0.73 & 217 \\
\hline 1 & 62500 & 100 & 35 & 42 & 1.05 & 218 \\
\hline 1 & 50625 & 110 & 36.3 & 48.2 & 1.18 & 222 \\
\hline 1 & 40000 & 142 & 34.7 & 110 & 0.18 & 229 \\
\hline 1 & 40000 & 82 & 39.7 & 46 & 1.54 & 230 \\
\hline 1 & 62500 & 75 & 45 & 100 & 1 & 234 \\
\hline 1 & 40000 & 142 & 30.3 & 45 & 0.47 & 237 \\
\hline 1 & 62500 & 100 & 29 & 42 & 1.67 & 240 \\
\hline 1 & 50625 & 110 & 36.3 & 48.2 & 2.15 & 246 \\
\hline 1 & 50625 & 110 & 36.3 & 48.2 & 3 & 248 \\
\hline 1 & 40000 & 129 & 39 & 48 & 0.92 & 248 \\
\hline 1 & 62500 & 100 & 40 & 42 & 1.18 & 249 \\
\hline 1 & 62500 & 120 & 28.9 & 34 & 1.46 & 260 \\
\hline 1 & 40000 & 142 & 46.6 & 45 & 0.47 & 271 \\
\hline 1 & 62500 & 100 & 71 & 42 & 1.18 & 275 \\
\hline 1 & 40000 & 142 & 29.6 & 110 & 0.43 & 317 \\
\hline 1 & 90000 & 134 & 34.3 & 48.2 & 0.71 & 329 \\
\hline 1 & 90000 & 134 & 38.6 & 48.2 & 0.71 & 386 \\
\hline 1 & 202500 & 134 & 44.9 & 48.2 & 0.71 & 400 \\
\hline 1 & 90000 & 131 & 38.6 & 48.1 & 1.56 & 431 \\
\hline 1 & 90000 & 131 & 37.5 & 64.9 & 1.21 & 438 \\
\hline 1 & 90000 & 131 & 32.4 & 48.1 & 1.56 & 451 \\
\hline 1 & 202500 & 131 & 32.4 & 48.1 & 1.56 & 504 \\
\hline 1 & 202500 & 131 & 39.4 & 48.1 & 1.56 & 511 \\
\hline 1 & 90000 & 131 & 75.8 & 57.4 & 1.56 & 547 \\
\hline 1 & 90000 & 284 & 39.4 & 48.2 & 0.34 & 781 \\
\hline 1 & 90000 & 284 & 34.3 & 48.2 & 0.34 & 825 \\
\hline 1 & 202500 & 284 & 48.6 & 48.2 & 0.34 & 911 \\
\hline 1 & 202500 & 284 & 32.4 & 48.2 & 0.34 & 1020 \\
\hline 1 & 90000 & 281 & 29.6 & 48.1 & 0.73 & 1027 \\
\hline 1 & 90000 & 281 & 39.4 & 48.1 & 0.73 & 1071 \\
\hline
\end{tabular}

TABLE 4: Continued.

\begin{tabular}{|c|c|c|c|c|c|c|}
\hline$X_{1}$ & $\begin{array}{c}\mathbf{X}_{2} \\
\left(\mathrm{~mm}^{2}\right)\end{array}$ & $\begin{array}{c}\mathbf{X}_{\mathbf{3}} \\
(\mathrm{mm})\end{array}$ & $\begin{array}{c}\mathbf{X}_{\mathbf{4}} \\
(\mathrm{MPa})\end{array}$ & $\begin{array}{c}\mathbf{X}_{\mathbf{5}} \\
(\mathrm{GPa})\end{array}$ & $\begin{array}{l}\mathbf{X}_{6} \\
(\%)\end{array}$ & $\begin{array}{c}\mathbf{Y} \\
(\mathrm{kN})\end{array}$ \\
\hline 1 & 90000 & 281 & 46.7 & 48.1 & 0.73 & 1195 \\
\hline 1 & 90000 & 281 & 46.7 & 48.1 & 0.73 & 1195 \\
\hline 1 & 202500 & 281 & 29.6 & 48.1 & 0.73 & 1248 \\
\hline 1 & 90000 & 275 & 38.2 & 56.7 & 1.61 & 1492 \\
\hline 1 & 90000 & 275 & 75.8 & 56.7 & 1.61 & 1600 \\
\hline 2 & 17671 & 95 & 32.6 & 147.6 & 0.19 & 142 \\
\hline 2 & 41548 & 95 & 33.2 & 147.6 & 0.19 & 150 \\
\hline 2 & 5027 & 89 & 35.9 & 40.7 & 3.78 & 171 \\
\hline 2 & 17671 & 96 & 36.7 & 91.8 & 0.26 & 181 \\
\hline 2 & 41548 & 96 & 37.3 & 91.8 & 0.26 & 189 \\
\hline 2 & 17671 & 95 & 118 & 37.3 & 0.64 & 207 \\
\hline 2 & 5027 & 122 & 32.1 & 44.8 & 1.21 & 217 \\
\hline 2 & 17671 & 89 & 35.8 & 40.7 & 3.78 & 231 \\
\hline 2 & 17671 & 122 & 32.1 & 44.8 & 1.21 & 237 \\
\hline 2 & 17671 & 95 & 35.7 & 95 & 1.05 & 255 \\
\hline 2 & 41548 & 95 & 36.3 & 95 & 1.05 & 273 \\
\hline 2 & 41548 & 126 & 34.3 & 92 & 0.52 & 343 \\
\hline 2 & 17671 & 126 & 33.8 & 92 & 0.52 & 347 \\
\hline 3 & 150000 & 110 & 35.2 & 43 & 1.2 & 362 \\
\hline 3 & 150000 & 135 & 35.2 & 43 & 1.2 & 484 \\
\hline 3 & 150000 & 137 & 53.1 & 43 & 0.35 & 506 \\
\hline 3 & 150000 & 140 & 40.3 & 122 & 0.4 & 530 \\
\hline 3 & 37500 & 162 & 42 & 85 & 0.28 & 534 \\
\hline 3 & 150000 & 135 & 53.1 & 43 & 0.7 & 549 \\
\hline 3 & 37500 & 162 & 42 & 85 & 0.28 & 575 \\
\hline 3 & 37500 & 162 & 42 & 85 & 0.28 & 584 \\
\hline 3 & 37500 & 162 & 42 & 85 & 0.28 & 622 \\
\hline 3 & 150750 & 165 & 49.6 & 122.5 & 0.35 & 674 \\
\hline 3 & 37500 & 162 & 42 & 85 & 0.28 & 698 \\
\hline 3 & 150000 & 135 & 64.8 & 43 & 1.2 & 704 \\
\hline 3 & 150250 & 159 & 44.3 & 38.5 & 1.99 & 712 \\
\hline 3 & 150500 & 156 & 49.2 & 46.5 & 1.21 & 732 \\
\hline 3 & 150000 & 159 & 49.6 & 44.6 & 1 & 740 \\
\hline 3 & 151000 & 165 & 44.3 & 122.5 & 0.69 & 799 \\
\hline 3 & 129375 & 165 & 59 & 147 & 0.57 & 1000 \\
\hline 3 & 129375 & 165 & 59 & 147 & 0.57 & 1200 \\
\hline 3 & 129375 & 165 & 59 & 147 & 0.57 & 1328 \\
\hline
\end{tabular}

\section{References}

[1] J. Bai, Advanced Fibre-Reinforced Polymer (FRP) Composites for Structural Applications, Woodhead Publishing Limited, 2013.

[2] Ç. Özes and N. Neşer, "Experimental study on steel to FRP bonded lap joints in marine applications," Advances in Materials Science and Engineering, vol. 2015, Article ID 164208, 6 pages, 2015.

[3] H. Fang, H. Shi, Y. Wang, Y. Qi, and W. Liu, "Experimental and theoretical study of sandwich panels with steel facesheets and 
GFRP core," Advances in Materials Science and Engineering, vol. 2016, Article ID 7159205, 12 pages, 2016.

[4] T. Ozbakkaloglu, J.-F. Chen, S. T. Smith, and J.-G. Dai, "Applications of fiber reinforced polymer composites," International Journal of Polymer Science, vol. 2016, Article ID 5804145, 2016.

[5] L. Bertolini, "Steel corrosion and service life of reinforced concrete structures," Structure and Infrastructure Engineering, vol. 4, no. 2, pp. 123-137, 2008.

[6] I. M. Metwally, "Prediction of punching shear capacities of twoway concrete slabs reinforced with FRP bars," HBRC Journal, vol. 9, no. 2, pp. 125-133, 2013.

[7] E.-K. Kim, H. Oh, and J. Sim, "Semiempirical methodology for estimating the service life of concrete deck panels strengthened with fiber-reinforced polymer," Mathematical Problems in Engineering, vol. 2014, Article ID 273693, 13 pages, 2014.

[8] S. M. Soleimani and N. Banthia, "Shear strengthening of RC beams using sprayed glass fiber reinforced polymer," Advances in Civil Engineering, vol. 2012, Article ID 635176, 20 pages, 2012.

[9] M. Hassan, E. A. Ahmed, and B. Benmokrane, "Punching shear behavior of two-way slabs reinforced with FRP shear reinforcement," Journal of Composites for Construction, vol. 19, no. 1, Article ID 04014030, 2015.

[10] M. Hassan, E. A. Ahmed, and B. Benmokrane, "Punching shear strength of glass fiber-reinforced polymer reinforced concrete flat slabs," Canadian Journal of Civil Engineering, vol. 40, no. 10, pp. 951-960, 2013.

[11] M. D. E. Teixeira, J. A. O. Barros, V. M. C. F. Cunha, B. N. Moraes-Neto, and A. Ventura-Gouveia, "Numerical simulation of the punching shear behaviour of self-compacting fibre reinforced flat slabs," Construction and Building Materials, vol. 74, pp. 25-36, 2015.

[12] K.-K. Choi, M. M. Reda Taha, H.-G. Park, and A. K. Maji, "Punching shear strength of interior concrete slab-column connections reinforced with steel fibers," Cement and Concrete Composites, vol. 29, no. 5, pp. 409-420, 2007.

[13] A. W. El-Ghandour, K. Pilakoutas, and P. Waldron, "Punching shear behavior of fiber reinforced polymers reinforced concrete flat slabs: experimental study," Journal of Composites for Construction, vol. 7, no. 3, pp. 258-265, 2003.

[14] A. A. Elshafey, E. Rizk, H. Marzouk, and M. R. Haddara, "Prediction of punching shear strength of two-way slabs," Engineering Structures, vol. 33, no. 5, pp. 1742-1753, 2011.

[15] M. A. W. Hassan, Punching shear behavior of concrete two-way slabs reinforced with Glass Fiber-Reinforced Polymer (GFRP) bars [Ph.D. thesis], University of Sherbrooke, 2013.

[16] A. Nanni, "Guide for the design and construction of concrete reinforced with FRP bars (ACI 440.1R-03)," in Proceedings of the Structures Congress and the 2005 Forensic Engineering Symposium-Metropolis and Beyond, pp. 1621-1626, New York, NY, USA, April 2005.

[17] D.-T. Vu and N.-D. Hoang, "Punching shear capacity estimation of FRP-reinforced concrete slabs using a hybrid machine learning approach," Structure and Infrastructure Engineering, vol. 12, no. 9, pp. 1153-1161, 2016.

[18] C.-C. Young, W.-C. Liu, and W.-L. Hsieh, "Predicting the water level fluctuation in an alpine lake using physically based, artificial neural network, and time series forecasting models," Mathematical Problems in Engineering, vol. 2015, Article ID 708204, 11 pages, 2015.

[19] T. Tran and N. Hoang, "Predicting colonization growth of algae on mortar surface with artificial neural network," Journal of Computing in Civil Engineering, vol. 30, no. 6, Article ID 04016030, 2016.

[20] A. P. Piotrowski, "Differential Evolution algorithms applied to Neural Network training suffer from stagnation," Applied Soft Computing Journal, vol. 21, pp. 382-406, 2014.

[21] M.-Y. Cheng and N.-D. Hoang, "Evaluating contractor financial status using a hybrid fuzzy instance based classifier: case study in the construction industry," IEEE Transactions on Engineering Management, vol. 62, no. 2, pp. 184-192, 2015.

[22] R. Storn and K. Price, "Differential evolution-a simple and efficient heuristic for global optimization over continuous spaces," Journal of Global Optimization, vol. 11, no. 4, pp. 341359, 1997.

[23] W. Hardle, Applied Nonparametric Regression, vol. 19 of Econometric Society Monographs, Cambridge University Press, Cambridge, UK, 1990.

[24] J. Klemela, Multivariate Nonparametric Regression and Visualization, Wiley Series in Computational Statistics, John Wiley \& Sons, Hoboken, NJ, USA, 2014.

[25] J. Wang, Y. Chen, T. Li, J. Lu, and L. Shen, "A residual-based kernel regression method for image denoising," Mathematical Problems in Engineering, vol. 2016, Article ID 5245948, 13 pages, 2016.

[26] D. Tien Bui, Q. P. Nguyen, N.-D. Hoang, and H. Klempe, "A novel fuzzy K-nearest neighbor inference model with differential evolution for spatial prediction of rainfall-induced shallow landslides in a tropical hilly area using GIS," Landslides, pp. 1-17, 2016.

[27] M.-Y. Cheng and N.-D. Hoang, "A Swarm-Optimized Fuzzy Instance-based Learning approach for predicting slope collapses in mountain roads," Knowledge-Based Systems, vol. 76, pp. 256-263, 2015.

[28] M. Ouassou, O. Kristiansen, J. G. O. Gjevestad, K. S. Jacobsen, and Y. L. Andalsvik, "Estimation of scintillation indices: a novel approach based on local kernel regression methods," International Journal of Navigation and Observation, vol. 2016, Article ID 3582176, 18 pages, 2016.

[29] A. W. El-Ghandour, K. Pilakoutas, and P. Waldron, "New approach for punching shear capacity prediction of fiber reinforced polymer reinforced concrete flat slabs," ACI Structural Journal, vol. 188, pp. 135-144, 1999.

[30] A. W. El-Ghandour, K. Pilakoutas, and P. Waldron, "Punching shear behavior and design of FRP RC flat slabs," in Proceedings of the International Workshop on Punching Shear Capacity of RC Slabs, Dedicated to Professor Sven Kinnunen, TRITA-BKN Bulletin 57, pp. 359-366, TRITA-BKN, Stockholm, Sweden, 2000.

[31] S. Matthys and L. Taerwe, "Concrete slabs reinforced with FRP grids. II: punching resistance," Journal of Composites for Construction, vol. 4, no. 3, pp. 154-161, 2000.

[32] C. E. Ospina, S. D. B. Alexander, and J. J. Roger Cheng, "Punching of two-way concrete slabs with fiber-reinforced polymer reinforcing bars or grids," ACI Structural Journal, vol. 100, no. 5, pp. 589-598, 2003.

[33] L. Nguyen-Minh and M. Rovňák, "Punching shear resistance of interior GFRP reinforced slab-column connections," Journal of Composites for Construction, vol. 17, no. 1, pp. 2-13, 2013.

[34] J.-S. Zhang, X.-F. Huang, and C.-H. Zhou, "An improved kernel regression method based on Taylor expansion," Applied Mathematics and Computation, vol. 193, no. 2, pp. 419-429, 2007. 
[35] Y.-Q. Yang, J.-S. Zhang, and X.-F. Huang, "Adaptive image enhancement algorithm combining kernel regression and local homogeneity," Mathematical Problems in Engineering, vol. 2010, Article ID 693532, 14 pages, 2010.

[36] K. Takezawa, Introduction to Nonparametric Regression, Wiley Series in Probability and Statistics, John Wiley \& Sons, Hoboken, NJ, USA, 2nd edition, 2006.

[37] E. A. Nadaraya, "On estimating regression," Theory of Probability and Its Applications, vol. 9, pp. 141-142, 1964.

[38] G. S. Watson, "Smooth regression analysis," The Indian Journal of Statistics. Series A, vol. 26, pp. 359-372, 1964.

[39] M. Wang, Z. Song, G. Dai, L. Peng, and C. Zheng, "Asteroids exploration trajectory optimal design with differential evolution based on mixed coding," International Journal of Distributed Sensor Networks, vol. 2015, Article ID 827987, 8 pages, 2015.

[40] M.-Y. Cheng and N.-D. Hoang, "Groutability estimation of grouting processes with microfine cements using an evolutionary instance-based learning approach," Journal of Computing in Civil Engineering, vol. 28, no. 4, Article ID 04014014, 2014.

[41] H. Peng, Z. Wu, P. Shao, and C. Deng, "Dichotomous binary differential evolution for knapsack problems," Mathematical Problems in Engineering, vol. 2016, Article ID 5732489, 12 pages, 2016.

[42] R. Landa Becerra and C. A. Coello, "Cultured differential evolution for constrained optimization," Computer Methods in Applied Mechanics and Engineering, vol. 195, no. 33-36, pp. 4303-4322, 2006.

[43] Z. Chen, F. Yan, X. Qiao, and Y. Zhao, "Sparse antenna array design for MIMO radar using multiobjective differential evolution," International Journal of Antennas and Propagation, vol. 2016, Article ID 1747843, 12 pages, 2016.

[44] S. K. Goudos, C. Kalialakis, and R. Mittra, "Evolutionary algorithms applied to antennas and propagation: a review of state of the art," International Journal of Antennas and Propagation, vol. 2016, Article ID 1010459, 12 pages, 2016.

[45] Q. Ding and G. Zheng, "The cellular differential evolution based on chaotic local search," Mathematical Problems in Engineering, vol. 2015, Article ID 128902, 15 pages, 2015.

[46] D. Tien Bui, B. T. Pham, Q. P. Nguyen, and N.-D. Hoang, "Spatial prediction of rainfall-induced shallow landslides using hybrid integration approach of Least-Squares Support Vector Machines and differential evolution optimization: a case study in Central Vietnam," International Journal of Digital Earth, vol. 9, no. 11, pp. 1077-1097, 2016.

[47] N.-D. Hoang, Q.-L. Nguyen, and Q.-N. Pham, "Optimizing construction project labor utilization using differential evolution: a comparative study of mutation strategies," Advances in Civil Engineering, vol. 2015, Article ID 108780, 8 pages, 2015.

[48] M.-Y. Cheng and D.-H. Tran, "Two-phase differential evolution for the multiobjective optimization of time-cost tradeoffs in resource-constrained construction projects," IEEE Transactions on Engineering Management, vol. 61, no. 3, pp. 450-461, 2014.

[49] A. Nasrollahi, M. Saffarzadeh, A. Isfahanian, and M. Ghayekhloo, "Application of a new binary harmony search algorithm in highway rehabilitation decision-making problems: a case study in Iran," Civil Engineering and Environmental Systems, vol. 32, no. 4, pp. 335-350, 2015.

[50] A. Kaveh and A. Nasrollahi, "A new hybrid meta-heuristic for structural design: ranked particles optimization," Structural Engineering and Mechanics, vol. 52, no. 2, pp. 405-426, 2014.
[51] A. S. Talaei, A. Nasrollahi, and M. Ghayekhloo, "An automated approach for optimal design of prestressed concrete slabs using PSOHS," KSCE Journal of Civil Engineering, pp. 1-10, 2016.

[52] M. H. Beale, M. T. Hagan, and H. B. Demuth, Neural Network Toolbox User's Guide, The MathWorks Inc, 2012.

[53] J. A. Freeman and D. M. Skapura, Neural Networks-Algorithms, Applications, and Programming Techniques, Addison-Wesley Publishing Company Inc, 1991.

[54] M. T. Hagan and M. B. Menhaj, "Training feedforward networks with the Marquardt algorithm," IEEE Transactions on Neural Networks, vol. 5, no. 6, pp. 989-993, 1994. 

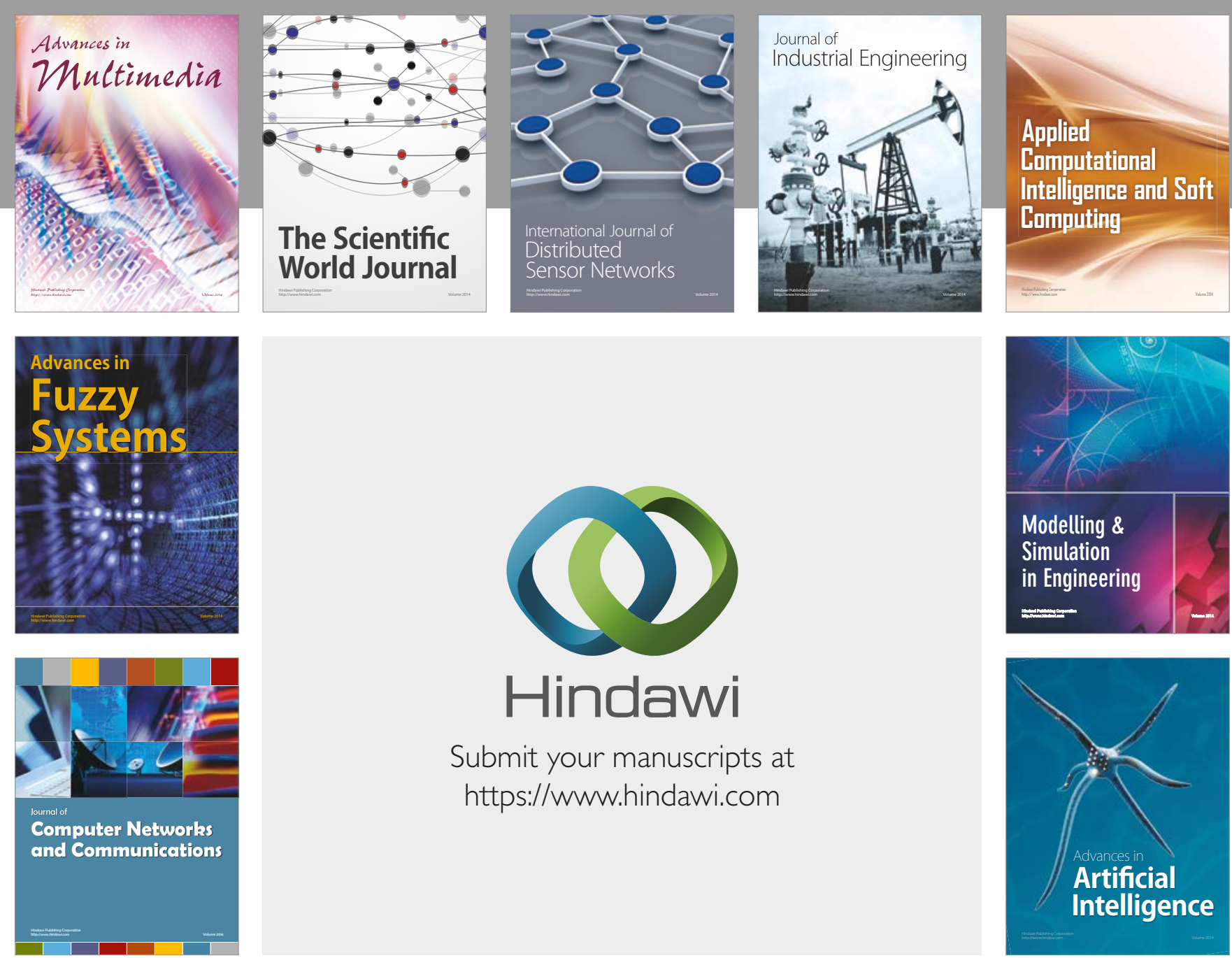

\section{Hindawi}

Submit your manuscripts at

https://www.hindawi.com
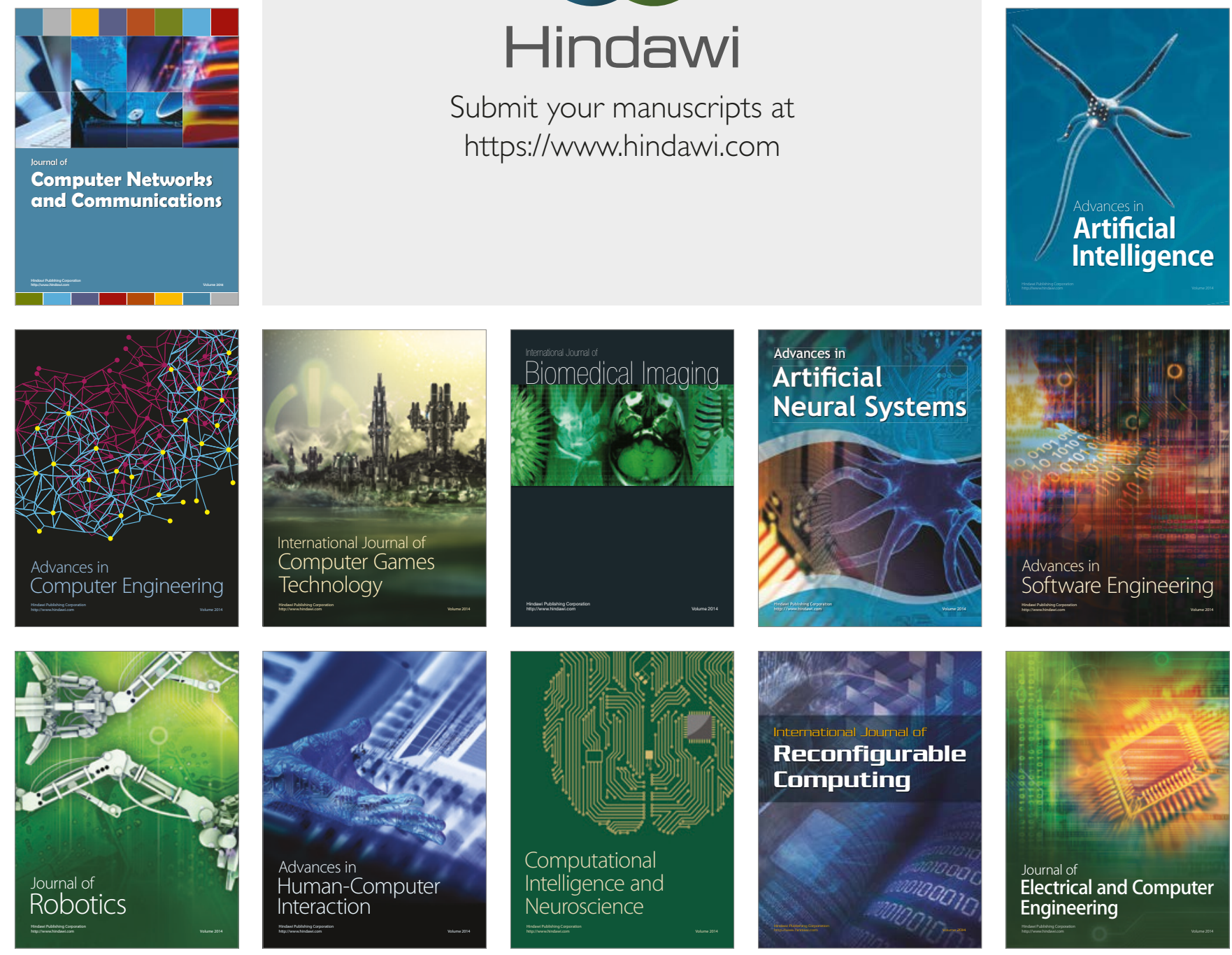